\title{
Characteristics of Men Who Perpetrate Intimate Partner Violence
}

\author{
Vijay Singh, MD, MPH, MS, Richard Tolman, PhD, Maureen Walton, MPH, PhD, \\ Stephen Chermack, PhD, and Rebecca Cunningham, MD
}

Purpose: Demographics, mental illness, substance use, and prior family violence are associated with perpetration of intimate partner violence (IPV) among male patient populations as well as court-based and community samples. However, few studies have identified health services use and physical symptoms associated with IPV perpetration among men. This study assesses the prevalence of IPV perpetration in a nationally representative sample of men and examines the associations of IPV perpetration with demographics, health services use, physical symptoms, mental illness, substance abuse, and prior family violence.

Methods: Data from the 2001 to 2003 National Comorbidity Survey-Replication was used to assess the prevalence of IPV perpetration among adult men. Bivariate and multivariate logistic regression determined associations of IPV perpetration with demographics, health services use, physical symptoms, mental health diagnoses, substance abuse/dependence, and prior family violence.

Results: The prevalence of male IPV perpetration is $19.2 \%$. Physical symptoms from irritable bowel syndrome (odds ratio [OR] 2.61; 95\% confidence interval $[\mathrm{CI}], 1.17-5.84)$ and insomnia (OR, 1.33; 95\% CI, 1.04-1.71), as well as substance abuse/dependence (OR, 1.76; 95\% CI, 1.09-2.85), were correlates of IPV perpetration in multivariate logistic regression analyses controlling for demographics and health services use. When prior family violence was added to the multivariate logistic regression model, only childhood family violence victimization $(\mathrm{OR}, 1.99 ; 95 \% \mathrm{CI}, 1.21-3.28)$ and witnessing childhood family violence (OR, 2.02; 95\% CI, 1.17-3.49) were associated with IPV perpetration.

Conclusions: Nearly 1 in 5 men in the United States reported lifetime IPV perpetration toward their current intimate partner. Physical symptoms from irritable bowel syndrome and insomnia, substance use disorders, and prior family violence are associated with IPV perpetration by men. Understanding these associations may aid primary care physicians in identifying male patients who perpetrate IPV. (J Am Board Fam Med 2014;27:661-668.)

Keywords: Domestic Violence, Men's Health, Substance Abuse

Most research and clinical guidelines on intimate partner violence (IPV) focus on screening and improving outcomes for women who are victims. Ap-

This article was externally peer reviewed.

Submitted 4 September 2013; revised 15 May 2014; accepted 6 June 2014.

From the Department of Emergency Medicine, Injury Center, and Institute for Healthcare Policy and Innovation (VS, RC), the Department of Family Medicine (VS), the School of Social Work (RT), and the Department of Psychiatry (MW, SC), University of Michigan, Ann Arbor.

Funding: This secondary analysis was developed with support from the Robert Wood Johnson Foundation and Veterans Affairs Clinical Scholars Program. Additional support was provided by the Centers for Disease Control and Prevention (grant 5R49CE002099) and the University of Michigan Injury Center. proximately 320,000 outpatient health care visits ${ }^{1}$ and 1200 deaths $^{2}$ due to IPV occur annually among women in the United States. In 2003, IPV resulted in an estimated $\$ 8.3$ billion spent on medical and mental health services and lost productivity. ${ }^{3}$ This amount does not include court costs, district and

Prior presentation: An earlier version of this paper was presented at the American Public Health Association Annual Meeting in Philadelphia, PA on November 10, 2009, and the Joint Annual Meeting of the Safe States Alliance, SAVIR, and CDC Core I \& II State Injury Grantees in Coralville, IA on April 6, 2011.

Conflict of interest: none declared.

Corresponding author: Vijay Singh, MD, MPH, MS, 2800 Plymouth Road, Suite B10-G080, Ann Arbor, MI 481092800 (E-mail: vijaysin@umich.edu). 
defense attorneys, or law enforcement. The Joint Commission on Accreditation of Health care Organizations mandates clinicians screen patients for IPV in all health care settings. ${ }^{4}$ In addition, the US Preventive Services Task Force recently recommended IPV screening and intervention services for asymptomatic women aged 14 to $46 .^{5}$

Studies have identified male patients, ${ }^{6-9}$ in addition to men in court-based ${ }^{10}$ and community ${ }^{11}$ samples, who perpetrate IPV against their female partners. One important reason for this focus on IPV perpetrated by men is gender differences in IPV injury and harm. While $14.8 \%$ of women experience an injury due to IPV during their lifetime, only $4 \%$ of men are injured from IPV. ${ }^{12}$ Demographic associations with IPV perpetration include young age, ${ }^{6}$ low educational attainment, ${ }^{6}$ and low income. ${ }^{7}$ History of violence including witnessing parental IPV and child abuse victimization are associated with IPV. ${ }^{7}$ Mental illness, including depression $^{7,8}$ and posttraumatic stress disorder (PTSD), ${ }^{9}$ and substance use disorders, including alcohol abuse $^{8,9}$ and illicit drug abuse, ${ }^{7-9}$ are all associated with IPV. To address IPV perpetration in primary care settings, an expert opinion-based clinical guideline outlines steps to identify male IPV perpetration, assess safety, and make referrals to mental health and substance use treatment programs, as well as community batterer treatment programs. ${ }^{13}$

Few studies have investigated physical symptoms and health services use among men who perpetrate IPV. Chronic pain from any source was recently shown to be associated with IPV perpetration by male primary care patients. ${ }^{9}$ No study to date has assessed associations between men who perpetrate IPV and specific pain conditions or physical symptoms such as those from irritable bowel syndrome (IBS) and insomnia. Physical symptoms such as those from IBS ${ }^{14}$ and insomnia ${ }^{15}$ have been demonstrated in IPV victims, and because many IPV victims and perpetrators have a history of child abuse or witnessing parental IPV, ${ }^{7}$ male IPV perpetrators may exhibit physical symptoms similar to symptoms exhibited by those who are IPV victims. Regarding health services use, only 2 small studies have assessed the primary care and emergency department (ED) utilization of male IPV perpetrators. Of men in one batterer treatment program, $63 \%$ had health visits for physical problems $^{16}$; in a separate program $44 \%$ reported receiving care in the ED. ${ }^{17}$ Understanding IPV perpetrators' health services use can uncover opportunities to identify these men.

This study's objectives are to (1) describe the prevalence of IPV perpetration by men in a nationally representative sample and (2) describe the associations of male IPV perpetration with demographics, health services use, physical symptoms, mental illness, substance use, and prior family violence. These objectives may lead to more inquiry about the relationship between IPV perpetration, physical symptoms, and health services use.

\section{Methods \\ Sampling}

We analyzed the 2001 to 2003 National Comorbidity Survey-Replication (NCS-R), which used a nationally representative, multistage, clustered sample of households. This probability sampling obtained respondents ages 18 or older living in the noninstitutionalized civilian household population of the United States. ${ }^{18}$ Harvard Medical School and the University of Michigan provided institutional review board approval for the original data collection. ${ }^{18}$ The University of Michigan institutional review board determined this current study to be not regulated because it is research using a publicly available data set.

We selected potential IPV perpetration risk domains based on prior findings ${ }^{6-9}$ and included demographics (age, race, education, health insurance, employment, and income); mental illness (depression, posttraumatic stress, panic, and anxiety disorders); substance use history (abuse or dependence of alcohol or drugs); and prior family violence (IPV victimization from age 21 , physical abuse as a child, and exposure to parental IPV). We selected physical symptoms or health conditions of chronic pain, epilepsy or seizures, injury, and headaches, which may cause men to seek primary care and have been associated with IPV perpetration in previous work. ${ }^{9}$ We chose IBS and insomnia symptoms given their association with IPV victimization. ${ }^{14,15}$ For relevance to identification in clinical settings, we selected health services use measures that can describe primary care and ED utilization.

\section{Intimate Partner Violence}

Physical abuse measures from the Conflict Tactics Scale $^{19}$ were adapted in the NCS-R to construct the IPV perpetration variable. In brief, the dependent variable IPV perpetration ${ }^{20}$ was assessed by 
asking: "Over the course of your relationship, how often have you ever done any of these things (pushed, grabbed, or shoved; threw something; slapped or hit; kicked, bit, or hit with a fist; beat up; choked; burned or scalded; threatened with a knife or gun) to your current spouse/partner?" Responses included often, sometimes, rarely, or never. We dichotomized responses into any/none.

\section{Experience with Violence as a Child}

The Conflict Tactics Scale measures of perpetration of parent-to-child physical abuse ${ }^{21}$ were adapted for experience with violence as a child. Victim of childhood family violence was measured by the question: "When you were growing up, how often did someone in your household do any of these things (push, grab, or shove; throw something; slap or hit) to you?" Responses were often, sometimes, rarely, or never. Witnessed childhood family violence was assessed by asking: "How often did (your parents/the people who raised you) do any of these things (push, grab, or shove; throw something; slap or hit) to each other while you were growing up?" Responses were often, sometimes, rarely, or never. We dichotomized responses into any/none.

\section{Demographics}

We dichotomized race between non-Hispanic white and all other race and ethnicity categories. We dichotomized education at high school completion. We dichotomized health insurance and employment as any/none. We divided income in dollars by $\$ 10,000$ increments.

\section{Health Services Use and Physical Symptoms or Health Conditions}

We assessed having a regular medical doctor for routine care and $\geq 1$ outpatient or ED visit per year. Responses were dichotomized. Men were asked whether they ever had chronic pain from arthritis, back or neck problems, or any other source; epilepsy or seizures; or injury. IBS symptom was measured by the question, "Have you ever had a period lasting 12 months or longer when at least 1 week each month you had frequent pain or discomfort in your stomach or lower abdomen that was relieved when you had a bowel movement?" We dichotomized IBS symptoms into yes/no. Insomnia symptoms were measured by the question, "How often do you have trouble getting to sleep or staying asleep?" Headache symptoms were measured by the question, "How often are you both- ered by headaches or pains in the head?" The response set for insomnia and headache symptoms were nearly all the time (1), pretty often (2), not very much (3), and never (4). We analyzed insomnia and headache responses using a mean frequency of positive report of symptoms.

\section{Substance Use and Mental Health Diagnoses}

Constructed variables using Diagnostic and Statistical Manual of Mental Disorders IV criteria $^{22}$ assessed the following lifetime diagnoses: substance abuse/ dependence (defined as alcohol abuse or dependence or drug abuse or dependence), major depressive disorder, PTSD, and anxiety disorders (defined as panic disorder or generalized anxiety disorder). ${ }^{20}$ We chose these mental health variables because of IPV perpetration associations in previous research. ${ }^{8,20}$ We dichotomized the responses.

\section{Statistical Analysis}

We conducted data analyses using STATA/SE software version 11 for Windows (StataCorp., College Station, TX). We analyzed age and income demographic variables, as well as insomnia and headache symptom physical health conditions, as continuous variables. Survey weights were applied in all analyses. First, we describe IPV perpetration among married or cohabiting men $\geq 21$ years old. Second, we present demographic characteristics of and prior family violence among the full sample, as well as univariate characteristics of patients who do and do not perpetrate IPV (Table 1). We also present bivariate logistic regression associations evaluating participants who do and do not perpetrate IPV (Table 1). We chose multivariate logistic regression models (Table 2) based on which bivariate associations were significant and theoretically grounded while adjusting for demographics and health insurance. The first model included only demographics, health services use, and physical symptoms because most primary care physicians do not assess Diagnostic and Statistical Manual of Mental Disorders-based substance use diagnoses, nor do they routinely obtain history of victimization from or witnessing childhood family violence. The second model added substance abuse and dependence to evaluate whether the associations with physical symptoms remained significant. The third model added prior family violence to understand the relationship of substance abuse and history of family violence to IPV. No multicollinearity of variables was observed in any of the 3 models. 
Table 1. Demographics and Prevalence of Prior Family Violence and Bivariate Analyses Comparing Intimate Partner Violence (IPV) Perpetration and Nonperpetration Among Married or Cohabiting Adult Men $(\mathrm{N}=530)$

\begin{tabular}{|c|c|c|c|c|}
\hline Variable & $\begin{array}{l}\text { Full sample } \\
(\mathrm{N}=530)\end{array}$ & $\begin{array}{l}\text { No IPV Perpetration } \\
\quad(\mathrm{n}=428)\end{array}$ & $\begin{array}{l}\text { IPV perpetration } \\
(\mathrm{n}=102)\end{array}$ & $\begin{array}{l}\text { IPV Perpetration, OR* } \\
(95 \% \mathrm{CI})\end{array}$ \\
\hline \multicolumn{5}{|l|}{ Demographics } \\
\hline Mean age, years (SD) & $42.6(13.1)$ & $43.0(13.4)$ & $41.0(11.8)$ & $0.98(0.96-1.00)$ \\
\hline Non-Hispanic white race & $415(78.3)$ & $339(79.2)$ & $76(74.5)$ & $0.77(0.46-1.27)$ \\
\hline Education beyond high school & $298(56.2)$ & $248(57.9)$ & $50(49)$ & $0.70(0.45-1.08)$ \\
\hline Health insurance & $383(72.3)$ & $311(72.7)$ & $72(70.6)$ & $0.90(0.56-1.45)$ \\
\hline Employed & $444(83.8)$ & $358(83.6)$ & $86(84.3)$ & $1.05(0.58-1.90)$ \\
\hline $\begin{array}{l}\text { Mean income, in tens of thousands } \\
\$(\text { SD) }\end{array}$ & $7.7(4.9)$ & $7.9(5.0)$ & $7.2(4.7)$ & $0.97(0.93-1.02)$ \\
\hline \multicolumn{5}{|l|}{ Health services use } \\
\hline Regular doctor for routine care & - & $324(75.7)$ & $66(64.7)$ & $0.59(0.37-0.93)^{\dagger}$ \\
\hline$\geq 1$ Routine health visit in past year & - & $266(62.1)$ & $54(52.9)$ & $0.69(0.44-1.06)$ \\
\hline$\geq 1$ Emergency room visit in past year & - & $115(26.9)$ & $32(31.1)$ & $1.24(0.78-1.99)$ \\
\hline \multicolumn{5}{|l|}{ Physical symptoms or health conditions } \\
\hline Chronic pain from arthritis & - & $88(20.6)$ & $23(22.5)$ & $1.12(0.67-1.89)$ \\
\hline Chronic back or neck pain & - & $130(30.4)$ & $31(30.4)$ & $1.00(0.63-1.60)$ \\
\hline Chronic pain from other sources & - & $39(9.1)$ & $14(13.7)$ & $1.59(0.83-3.05)$ \\
\hline Epilepsy or seizures & - & $9(2.1)$ & $1(1.0)$ & $0.46(0.06-3.68)$ \\
\hline Injury or poisoning in past year & - & $42(9.8)$ & $10(9.8)$ & $1.00(0.48-2.06)$ \\
\hline Irritable bowel syndrome symptoms & - & $18(4.2)$ & $12(11.8)$ & $3.04(1.41-6.53)^{\ddagger}$ \\
\hline Insomnia symptoms, mean (SD) & - & $1.98(0.85)$ & $2.25(0.89)$ & $1.42(1.11-1.81)^{\ddagger}$ \\
\hline Headache symptoms, mean (SD) & - & $1.78(0.65)$ & $1.89(0.72)$ & $1.27(0.93-1.75)$ \\
\hline \multicolumn{5}{|l|}{ Mental health diagnoses, DSM-IV criteria } \\
\hline Substance abuse/dependence ${ }^{\S}$ & - & $96(22.4)$ & $39(38.2)$ & $2.14(1.35-3.39)^{\ddagger}$ \\
\hline Major depressive disorder & - & $78(18.2)$ & $19(18.6)$ & $1.03(0.59-1.79)$ \\
\hline Posttraumatic stress disorder & - & $20(4.67)$ & $5(5.0)$ & $1.05(0.39-2.87)$ \\
\hline Anxiety disorders $\|^{\|}$ & - & $46(10.7)$ & $15(14.0)$ & $1.43(0.76-2.68)$ \\
\hline \multicolumn{5}{|l|}{ Prior family violence } \\
\hline Victim of childhood family violence ${ }^{\mathbb{I}}$ & $234(44.2)$ & $170(39.7)$ & $64(63.4)$ & $2.63(1.68-4.11)^{* *}$ \\
\hline Witnessed childhood family violence ${ }^{\dagger \dagger}$ & $97(18.4)$ & $62(14.6)$ & $35(35)$ & $3.16(1.93-5.17)^{* *}$ \\
\hline
\end{tabular}

Data are n (weighted \%) unless otherwise indicated. IPV perpetration includes having pushed, grabbed, or shoved; threw something; slapped or hit; kicked, bit, or hit with a fist; beat up; choked; burned or scalded; and threatened their partner with a knife or gun.

${ }^{*}$ The odds ratio reference category is no IPV perpetration.

${ }^{\dagger} P<.05$.

${ }^{\ddagger} P<.01$.

${ }^{\$}$ Alcohol abuse, alcohol dependence, drug abuse, or drug dependence.

"Panic disorder or generalized anxiety disorder.

${ }^{\mathbb{N}} \mathrm{N}=529$ because only 529 randomly assigned men received both the IPV perpetration and victim of childhood family violence questions. ${ }^{* *} P<.001$.

${ }^{+\dagger} \mathrm{N}=526$ because only 526 randomly assigned men received both the IPV perpetration and witnessed childhood family violence questions.

\section{Results}

\section{Overall Sample}

The original NCS-R sample was composed of 4139 men, of whom we analyzed a subset of 530 men who received questions on IPV perpetration, physical symptoms, and health conditions. In this nationally representative sample of $530 \mathrm{men}$, the prevalence of IPV perpetration was $19.2 \%$ ( $\mathrm{n}=$ 102). Demographic characteristics of these men (Table 1) include an average age 42.6 years, $78.3 \%$
non-Hispanic white, $56.2 \%$ educated beyond high school, $83.8 \%$ employed, and $72.3 \%$ insured.

\section{Bivariate Analysis of Male IPV Perpetrators and Nonperpetrators}

The bivariate logistic regression analyses (Table 1) found that no demographic variable including income, education, or race was associated with IPV perpetration. Of health services use variables, men who reported having a regular doctor for routine 
Table 2. Multivariate Logistic Regression Showing Correlates of Any Intimate Partner Violence (IPV) Perpetration Among Adult Married and Cohabiting Men

\begin{tabular}{|c|c|c|c|}
\hline \multirow[b]{2}{*}{ Characteristics } & \multicolumn{3}{|c|}{ IPV Perpetration } \\
\hline & Model $1(\mathrm{n}=530)$ & Model $2(\mathrm{n}=530)$ & Model 3* $(\mathrm{n}=526)$ \\
\hline \multicolumn{4}{|l|}{ Demographics } \\
\hline Age, years (in decades) & $1.00(0.98-1.02)$ & $1.00(0.98-1.02))$ & $1.00(0.98-1.02)$ \\
\hline Non-Hispanic white race vs. other & $0.80(0.48-1.35)$ & $0.79(0.47-1.34)$ & $0.80(0.46-1.37)$ \\
\hline Employed & $1.19(0.60-2.36)$ & $1.20(0.60-2.39)$ & $1.09(0.53-2.22)$ \\
\hline Education beyond high school & $0.80(0.50-1.29)$ & $0.84(0.52-1.36)$ & $0.90(0.55-1.49)$ \\
\hline Income, $\$$ (in tens of thousands) & $0.99(0.94-1.05)$ & $0.99(0.94-1.05)$ & $0.98(0.93-1.03)$ \\
\hline \multicolumn{4}{|l|}{ Health services use } \\
\hline Regular doctor for routine care & $0.67(0.41-1.09)$ & $0.72(0.43-1.18)$ & $0.73(0.44-1.23)$ \\
\hline \multicolumn{4}{|l|}{ Physical symptoms } \\
\hline Irritable bowel syndrome symptoms & $2.80(1.26-6.21)^{\dagger}$ & $2.61(1.17-5.84)^{\dagger}$ & $2.19(0.95-5.01)$ \\
\hline Insomnia symptoms & $1.38(1.08-1.77)^{\dagger}$ & $1.33(1.04-1.71)^{\dagger}$ & $1.29(0.99-1.67)$ \\
\hline \multicolumn{4}{|l|}{ Mental health diagnoses, DSM-IV criteria } \\
\hline Substance abuse/dependence ${ }^{\ddagger}$ & & $1.76(1.09-2.85)^{\dagger}$ & $1.60(0.97-2.66)$ \\
\hline \multicolumn{4}{|l|}{ Prior family violence } \\
\hline Victim of childhood family violence & & & $1.99(1.21-3.28)^{\S}$ \\
\hline Witnessed childhood family violence & & & $2.02(1.17-3.49)^{\dagger}$ \\
\hline
\end{tabular}

Data are odds ratios (95\% confidence intervals). No IPV perpetration is odds ratio reference category. IPV perpetration includes having pushed; grabbed or shoved; threw something; slapped or hit; kicked, bit, or hit with a fist; beat up; choked; burned or scalded; or threatened their partner with a knife or gun. Model 1 includes only demographics, health services use, and physical symptoms that primary care physicians routinely assess. Model 2 added substance abuse and dependence to evaluate whether the associations with physical symptoms remained significant. Model 3 added prior family violence to understand the relationship of IPV to substance abuse and history of family violence.

${ }^{*}$ Model 3 cohort was $\mathrm{n}=526$ because only 526 randomly assigned men received both the IPV perpetration and prior family violence questions.

${ }^{\dagger} P<.05$.

${ }^{\ddagger}$ Alcohol abuse, alcohol dependence, drug abuse, or drug dependence.

${ }^{\$} P<.01$.

care had decreased odds of IPV perpetration. Of physical symptoms or health conditions evaluated, IBS and insomnia symptoms had increased odds (odds ratio [OR] of 3.0 and 1.4, respectively) of IPV perpetration. For mental health diagnoses, substance abuse/dependence was significantly associated with IPV perpetration, but depression, PTSD, and anxiety disorders were not. Prior family violence-both victimization from or witnessing childhood family violence-were strongly associated with IPV perpetration.

\section{Multivariate Logistic Regression Analysis Showing Correlates of Male IPV Perpetration}

Multivariate logistic regression analyses (Table 2) found IBS (OR, 2.80; 95\% confidence interval [CI], 1.26-6.21) and insomnia symptoms (OR, 1.38; 95\% CI, 1.08-1.77) were associated with IPV perpetration in model 1 . When adding substance abuse or dependence to the first model, as might occur when a primary care physician obtains a more in-depth history, IBS (OR, 2.6; 95\% CI, 1.17-5.84) and insomnia (OR, 1.33; 95\% CI, 1.04-1.71) symptoms remained significant; substance abuse/ dependence (OR, 1.76; 95\% CI, 1.09-2.85) also was associated with IPV perpetration. In model 3, however, in which a history of family violence was assessed, only victim of childhood family violence (OR, 1.99; 95\% CI, 1.21-3.28) and witnessing childhood family violence (OR, 2.02; 95\% CI, 1.17-3.49) remained associated with IPV perpetration.

\section{Discussion}

Over their current relationship, nearly 1 in 5 men in the United States report perpetrating IPV toward an intimate partner. This IPV perpetration prevalence is similar to other national estimates. ${ }^{20}$

No other nationally representative sample of men has assessed routine health and ED visits 
among those who do and do not perpetrate IPV. This data found more than half the men who perpetrate IPV reported at least one primary care visit in the past year, and nearly one-third noted at least one ED visit. This finding suggests male IPV perpetrators seek routine medical services, giving primary care providers the opportunity to identify men's aggressive behavior and potentially intervene. Although having a regular doctor for routine care was significant in the bivariate analysis, when controlling for IBS and insomnia symptoms this bivariate association was not significant in the multivariate analysis. This finding suggests that the health services use by IPV perpetrators may be related to physical symptoms.

Almost $12 \%$ of men who report perpetrating IPV also note IBS symptoms, and these men experience insomnia symptoms pretty often (mean score, 2.25). Patients might initiate a discussion of these physical symptoms during a routine visit, or the symptoms may be uncovered as part of a general review of systems during a comprehensive visit. The association of IPV perpetration with IBS and insomnia symptoms has not previously been noted in a nationally representative sample.

The second model showed that including substance use in the analysis does not eliminate the association of physical symptoms with IPV. This novel finding for men who perpetrate IPV could serve as a reminder for health care providers to inquire more in depth about substance use among patients with IBS and insomnia symptoms. Prior research has shown IBS to be associated with childhood sexual assault, ${ }^{23}$ substance use including alcohol, and insomnia. ${ }^{24}$ Future work is needed to understand the relationship of substance use, insomnia, IBS, and IPV perpetration. However, given that the previously described clinical guideline already outlines an approach to identifying IPV perpetration among male patients, ${ }^{13}$ this study can provide further direction for primary care providers to find cases of IPV perpetration based on physical symptoms and substance use indicators. In addition, when compared with primary care studies, ${ }^{7-9}$ this study showed similar findings of substance use associations with IPV perpetration. In contrast, primary care studies have shown depression and PTSD to be associated with IPV perpetration, ${ }^{7-9}$ although this study did not.

When prior family violence was considered in the third model, no other factors were significant.
This finding supports previous reports that IPV is intergenerational, with IPV perpetration associated with victimization from or witnessing childhood family violence. ${ }^{25}$ This suggests that prior family violence, which is well identified in the literature as being related to perpetration of IPV as an adult, may be interrelated with insomnia and IBS symptoms, as well as substance abuse/dependence. Primary care providers who are evaluating symptoms of insomnia and IBS should consider both IPV perpetration as well as a history of prior family violence.

The implications of this study for primary care are that while screening for IPV perpetration is not routine, it is important for understanding the above associations and sharing available resources. The US Preventive Services Task Force IPV victimization screening recommendation does not comment on identifying or responding to men who are victims or perpetrators of IPV. ${ }^{5}$ However, the American Medical Association encourages routine screening for IPV victimization and perpetration among men and women, in addition to inquiry about history of family violence and substance use. ${ }^{26}$ This study provides empiric support for American Medical Association recommendations. Our results also show how nearly 2 of 3 male IPV perpetrators are victims of childhood family violence. Primary care providers can maintain compassion toward male patients who perpetrate IPV by knowing that these patients might also have experienced childhood abuse.

There were several limitations to our study. Because the NCS-R collects cross-sectional data, only associations with, and not causation of, IPV perpetration can be determined. The men's responses were self-report only, with no independent validation of responses outside of partner report. In addition, only physical violence was assessed in the NCS-R, so the prevalence measures do not capture rates of verbal, emotional, and sexual forms of IPV or childhood family violence victimization or exposure. Further, the majority of the sample was nonHispanic white; additional examination of these issues is needed for minority groups.

These limitations notwithstanding, IPV perpetration by men is a large public health problem. When primary care providers encounter men with IBS or insomnia symptoms, physicians can consider assessing for substance use, childhood family violence, and IPV perpetration. If these men receive mental health, substance use, and/or IPV perpetra- 
tion referrals and interventions, physicians may prevent subsequent violence and injuries by these men to their partners. Such mental health, substance use, and IPV referrals and interventions could be integrated within primary care. Future IPV perpetration research needs to include mental health associations and understand what interventions are helpful.

\section{References}

1. Tjaden P, Thoennes N. Extent, nature, and consequences of intimate partner violence: findings from the National Violence Against Women Survey. Washington, DC: U.S. Department of Justice, Office of Justice Programs, National Institute of Justice; 2000.

2. Catalano, S. Intimate partner violence in the United States. Washington, DC: US Department of Justice, Bureau of Justice Statistics; 2007. Available from: http:// www.bjs.gov/content/pub/pdf/ipvus.pdf. Accessed May 12, 2014.

3. Max W, Rice DP, Finkelstein E, Bardwell RA, Leadbetter S. The economic toll of intimate partner violence against women in the United States. Violence Vict 2004;19:259-72.

4. Joint Commission on Accreditation of Healthcare Organizations. Accreditation Program Hospital: provision of care, treatment, and services. Washington, D.C.: Joint Commission on Accreditation of Healthcare Organizations; 2008.

5. Moyer VA. Screening for intimate partner violence and abuse of elderly and vulnerable adults: a U.S. Preventive Services Task Force recommendation statement. Ann Intern Med 2013;158:478-86.

6. Singh V. Academic men's health. Case studies in clinical practice: intimate partner violence perpetration. J Mens Health 2009;6:383-92.

7. Oriel KA, Fleming MF. Screening men for partner violence in a primary care setting: a new strategy for detecting domestic violence. J Fam Pract 1998;46: 493-8.

8. Rhodes KV, Houry D, Cerulli C, Straus H, Kaslow NJ, McNutt L. Intimate partner violence and comorbid mental health conditions among urban male patients. Ann Fam Med 2009;7:47-55.

9. Taft C, Schwartz S, Liebshutz JM. Intimate partner aggression perpetration in primary care chronic pain patients. Violence Vict 2010;25:649-61.

10. Basile KC, Hall JE, Walters ML. Expanding resource theory and feminist-informed theory to explain intimate partner violence perpetration by court-ordered men. Violence Against Women 2013; 19:848-80.

11. Desmarais SL, Reeves KA, Nicholls TL, Telford RP, Fiebert MS. Prevalence of physical violence in intimate relationships, part 2: rates of male and female perpetration. Partner Abuse 2012;3:170-98.

12. Black MC, Basile KC, Breiding MJ, et al. National Intimate Partner and Sexual Violence Survey (NISVS): 2010 summary report. Atlanta, GA: Centers for Disease Control and Prevention, National Center for Injury Prevention and Control, Division of Violence Prevention and Control; 2011.

13. Kimberg LS. Addressing intimate partner violence with male patients: a review and introduction of pilot guidelines. J Gen Intern Med 2008;23:2071-8.

14. Bonomi AE, Anderson ML, Reid RJ, Rivara FP, Carrell D, Thompson RS. Medical and psychosocial diagnoses in women with a history of intimate partner violence. Arch Intern Med 2009;169:1692-7.

15. Pigeon WR, Cerulli C, Richards H, He H, Perlis M, Caine E. Sleep disturbances and their association with mental health among women exposed to intimate partner violence. J Womens Health (Larchmt) 2011;20:1923-9.

16. Gerlock AA. Health impact of domestic violence. Issues Ment Health Nurs 1999;20:373-85.

17. Coben JH, Friedman DI. Health care use by perpetrators of domestic violence. J Emerg Med 2002;22: 313-7.

18. Kessler RC, Berglund P, Chiu WT, et al. The US National Comorbidity Survey Replication (NCS-R): design and field procedures. Int J Methods Psychiatr Res 2004;13:69-92.

19. Straus MA. Measuring intra-family conflict and violence: the Conflict Tactics Scales. J Marriage Fam 1979;41:75-88.

20. Kessler RC, Molnar BE, Feurer ID, Appelbaum M. Patterns and mental health predictors of domestic violence in the United States: results from the $\mathrm{Na}$ tional Comorbidity Survey. Int J Law Psychiatry 2001; 24:487-508.

21. Straus MA, Paschall MJ. Corporal punishment by mothers and development of children's cognitive ability: a longitudinal study of two nationally representative age cohorts. J Aggress Maltreat Trauma 2009; 18:459-83.

22. Kessler RC, Demler O, Frank RG, et al. Prevalence and treatment of mental disorders, 1990 to 2003. N Engl J Med 2005;352):2515-23.

23. Blanchard EB, Keefer L, Lackner JM, Galovski TE, Krasner S, Sykes MA. The role of childhood abuse in Axis I and Axis II psychiatric disorders and medical disorders of unknown origin among irritable bowel syndrome patients. J Psychosom Res 2004;56:431-6.

24. Lackner JM, Ma CX, Keefer L, et al. Type, rather than number, of mental and physical comorbidities 
increases the severity of symptoms in patients with irritable bowel syndrome. Clin Gastroenterol Hepatol 2013;11:1147-57.

25. Roberts AL, McLaughlin KA, Conron KJ, Koenen KC. Adulthood stressors, history of childhood adversity, and risk of perpetration of intimate partner violence. Am J Prev Med 2011;40:128-38.
26. AMA code of medical ethics. Opinion 2.02. Physicians' obligations in preventing, identifying, and treating violence and abuse. Chicago: American Medical Association; 2009. Available from: http://www.ama-assn.org/ $\mathrm{ama} / \mathrm{pub} / \mathrm{physician}$-resources/medical-ethics/codemedical-ethics/opinion202.page? Accessed July 22, 2014. 